\title{
Kinetic simulations of 3-D reconnection and magnetotail disruptions
}

\author{
P. L. Pritchett and F. V. Coroniti \\ Department of Physics and Astronomy, University of California, Los Angeles, CA 90095-1547, U.S.A.
}

(Received May 15, 2000; Revised August 14, 2000; Accepted October 12, 2000)

\begin{abstract}
The effects of a full 3-D geometry on collisionless reconnection are still basically unknown. Large scale 3-D particle-in-cell simulations are used to investigate the role of internal instabilities associated with the third dimension on the reconnection process and to examine the effect of boundary conditions which are imposed on a localized reconnection region by the large scale system. For an idealized current sheet of the Harris type the internal instabilities are found not to substantially alter the structure of the 2-D diffusion region. A convection electric field localized near midnight drives a magnetotail configuration to reconnect with the formation of a thin, electron dominated current sheet and a region 2 sense field-aligned current system in the reconnection region. These processes, however, do not appear to exhibit the characteristics associated with substorm onset.
\end{abstract}

\section{Introduction}

The conversion of energy stored in stressed magnetic fields into high speed plasma flows and thermal energy via magnetic reconnection is a fundamental plasma process that occurs in many plasma systems possessing magnetic shear. Sawteeth and disruptions in tokamaks, substorms in the terrestrial magnetotail, and solar flares are several phenomena which are believed to involve reconnection as a dominant, although not necessarily the only, dissipation process. These systems exhibit a certain commonality-magnetic energy accumulates on a relatively long time scale and is then released suddenly or explosively on nearly the Alfvén time scale.

This bimodal character (directly driven versus explosive) indicates that the understanding of reconnection must be pursued at two levels: (1) the detailed microphysics which breaks the frozen-in condition and allows plasma to slip through the magnetic field and the lines of force to reconnect, and (2) the effects of the boundary conditions which the large scale system imposes on the reconnection region and the back reaction on the global system of the reconnection-induced changes in the magnetic field and plasma flow state. The microphysics will, of course, be different in different systems (collisional versus collisionless), and the global boundary conditions are system specific. Nevertheless, the common explosive nature of reconnection suggests that a detailed understanding of these two aspects of reconnection in any one system is likely to contain valuable lessons and insights for the behavior of reconnection in other systems. In this paper we use particle-in-cell (PIC) simulations to address aspects of these twin issues.

Our present understanding of collisionless reconnection microphysics is largely based on two-dimensional (2-D) models. Under the GEM reconnection challenge program (Birn et al., 2001 and references therein), numerical simula-

Copy right (C) The Society of Geomagnetism and Earth, Planetary and Space Sciences (SGEPSS); The Seismological Society of Japan; The Volcanological Society of Japan; The Geodetic Society of Japan; The Japanese Society for Planetary Sciences. tions of a 2-D reconnection configuration have greatly clarified the microphysics by which inflowing electrons and ions decouple from the magnetic field in the diffusion region and then flow away from the neutral line along with the reconnected magnetic flux. The dynamics of the system in the diffusion region is linked to Hall physics and is characterized by a quadrupole out-of-plane magnetic field pattern (Sonnerup, 1979; Terasawa, 1983) and a very thin $\left(\sim c / \omega_{p e}\right)$ electron dominated current layer. A full 3-D geometry, however, allows for a much larger class of instabilities, and it is possible that the localized current layers observed in 2D could break up, which could significantly alter the reconnection rate and the structure of the discontinuities which develop outside of the dissipation region. To investigate these issues, we present in Section 3 the results of large scale 3-D PIC simulations of reconnection in an idealized current sheet.

Understanding the onset mechanism for substorms remains one of the preeminent problems in magnetospheric physics. Recent multi-spacecraft studies (e.g., Angelopoulos et al., 1997; Petrukovich et al., 1998; Lui et al., 1998) have provided clear evidence that substorm onset and its associated phenomena occur in a limited longitudinal range on the order of $3 \mathrm{R}_{\mathrm{E}}$ or less. These observations are inconsistent with the traditional 2-D view that onset involves a tearing instability along an extended neutral line. In an attempt to understand such localized disruption in the magnetotail, we present in Section 4 the results of 3-D PIC simulations of driven reconnection in a model tail configuration. A longitudinally localized convection electric field is applied in the lobes and at the inner (near-Earth) radial boundary. We discuss the resulting reconnection process, including the formation of an embedded thin current sheet and the generation of a field-aligned current system.

\section{Simulation Model}

The simulations to be discussed in this paper employ a 3-D PIC model which retains the full dynamics for both 
electrons and ions. As described in Pritchett et al. (1996, 1997), the electric and magnetic fields are obtained by integrating the time-dependent Maxwell equations forward in time using an explicit leapfrog scheme, and the relativistic particle equations of motion are likewise integrated in time using a leapfrog scheme. A Poisson correction is applied to the electric field to ensure that the current continuity equation remains valid for the charge and current densities interpolated onto the spatial grid.

The present simulations employ two alternative initial equilibrium configurations. The first is the generalized 2D Harris current sheet (Schindler, 1972; Birn et al., 1975; Lembège and Pellat, 1982) in which the 2-D magnetic fields are obtained from the vector potential $A_{0 y}(x, z)$ given by

$$
A_{0 y}(x, z)=L B_{0} \ln \{\cosh [F(x)(z / L)] / F(x)\} .
$$

Here, $F(x)$ is a slowly varying, but otherwise arbitrary, function. The $B_{x}$ field derived from (1) is

$$
B_{0 x}(x, z)=-B_{0} F(x) \tanh [F(x)(z / L)] .
$$

Thus the asymptotic $(|z| \rightarrow \infty)$ strength of the $B_{0 x}$ field is $B_{0} F(x)$ and the effective sheet thickness is $L / F(x)$. We
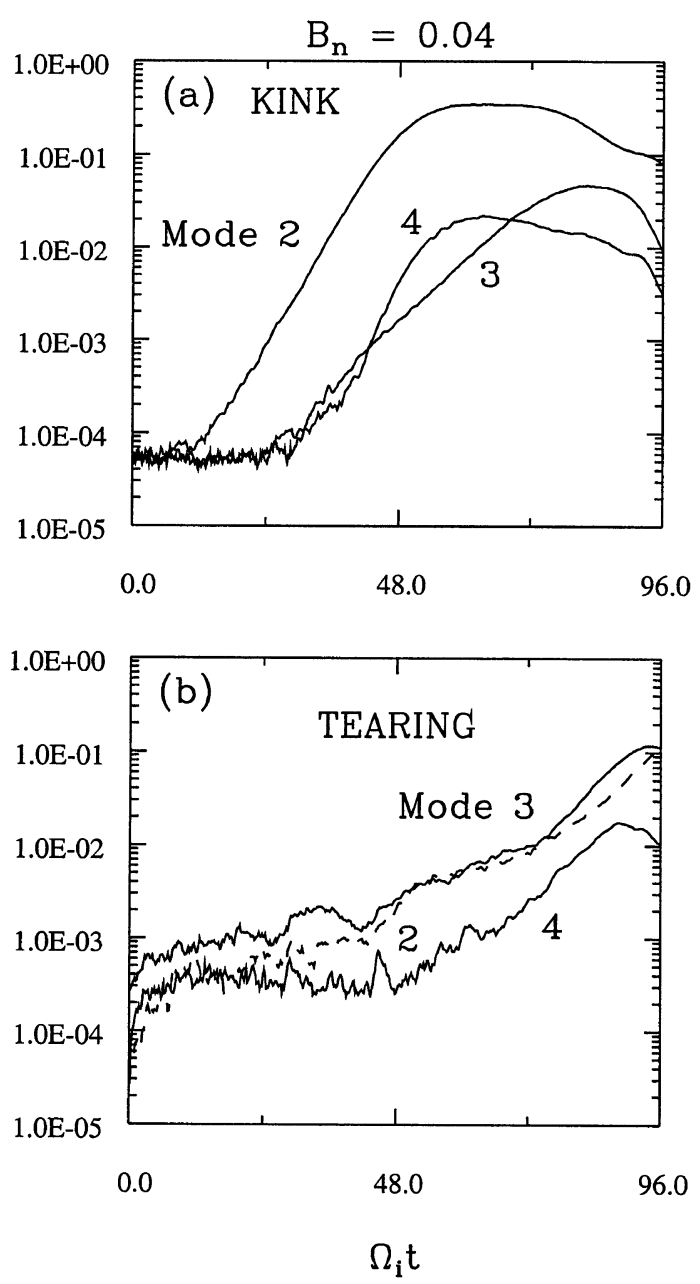

Fig. 1. Time histories of the absolute value squared of the ion density perturbations in a 3-D current sheet simulation with normal field $B_{n} / B_{0}=0.04$. The upper panel shows the pure kink modes $\left(k_{x}=0, k_{y}=2,3,4\right)$, while the lower panel shows the pure tearing modes $\left(k_{x}=2,3,4, k_{y}=0\right)$. shall use the quasi-parabolic form for $F(x)$ introduced by Lembège and Pellat (1982),

$$
F(x)=\exp (-\epsilon x / L)
$$

where $\epsilon \ll 1$ is a constant that determines the strength of the normal field $B_{z}$ on axis,

$$
B_{0 z}(x, 0)=\epsilon B_{0}
$$

The density is given by

$$
n(x, z)=n_{b}+n_{0} F^{2}(x) \operatorname{sech}^{2}[F(x)(z / L)],
$$

where $n_{b}$ represents a uniform background component.

The second configuration is more realistic for studies of magnetotail processes. Here the magnetic field is given by a superposition of a line (2-D) dipole and the current sheet equilibrium (1)-(3) with $\epsilon=0.1$. The ions are loaded initially according to the distribution (5), and then they are allowed to move under the influence of the combined magnetic fields. This leads to an initial earthward flow, which then stabilizes with the ion distribution experiencing no net force (Pritchett and Coroniti, 1995). The electrons are then initialized at the same spatial coordinates as the ions with a Maxwellian distribution with a temperature $T_{e 0}=T_{i 0} / 4$ and a local drift satisfying $v_{d e}=-\left(T_{e 0} / T_{i 0}\right) v_{d i}$.

Both initial configurations are independent of the $y$ coordinate (east-west in a magnetotail system), and thus it is possible to assume periodic boundary conditions in $y$. At the $z$ boundaries, which are located far away from the central current sheet, the perturbed components of the tangential electric field $\left(\delta E_{x}, \delta E_{y}\right)$ and the normal component of the magnetic field $\left(\delta B_{z}\right)$ are assumed to vanish, and particles striking the boundaries are specularly reflected. At the $x$ boundaries particles are free to exit from the system, and new particles are injected into the system based on a thermal distribution. For the fields "open" boundary conditions are assumed (Pritchett and Coroniti, 1998) whereby $\delta B_{z}$ and $\delta E_{z}$ are taken to vanish. In addition, for the second (magnetotail) configuration a localized convection electric field $E_{y}$

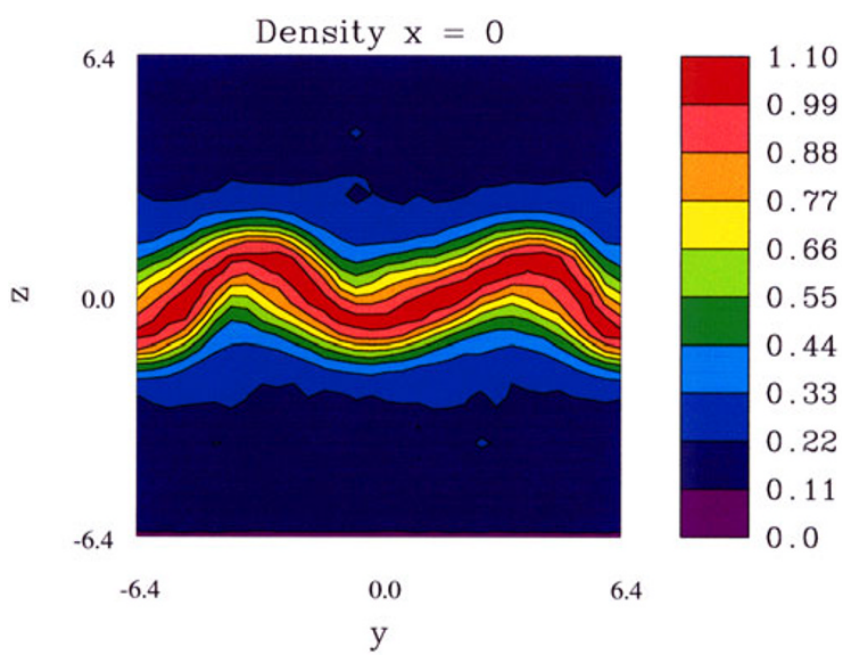

Fig. 2. Contour plot of the density in the $x, y$ plane at $x=0$ at time $\Omega_{i 0} t=64$ for the same simulation as in Fig. 1 . 


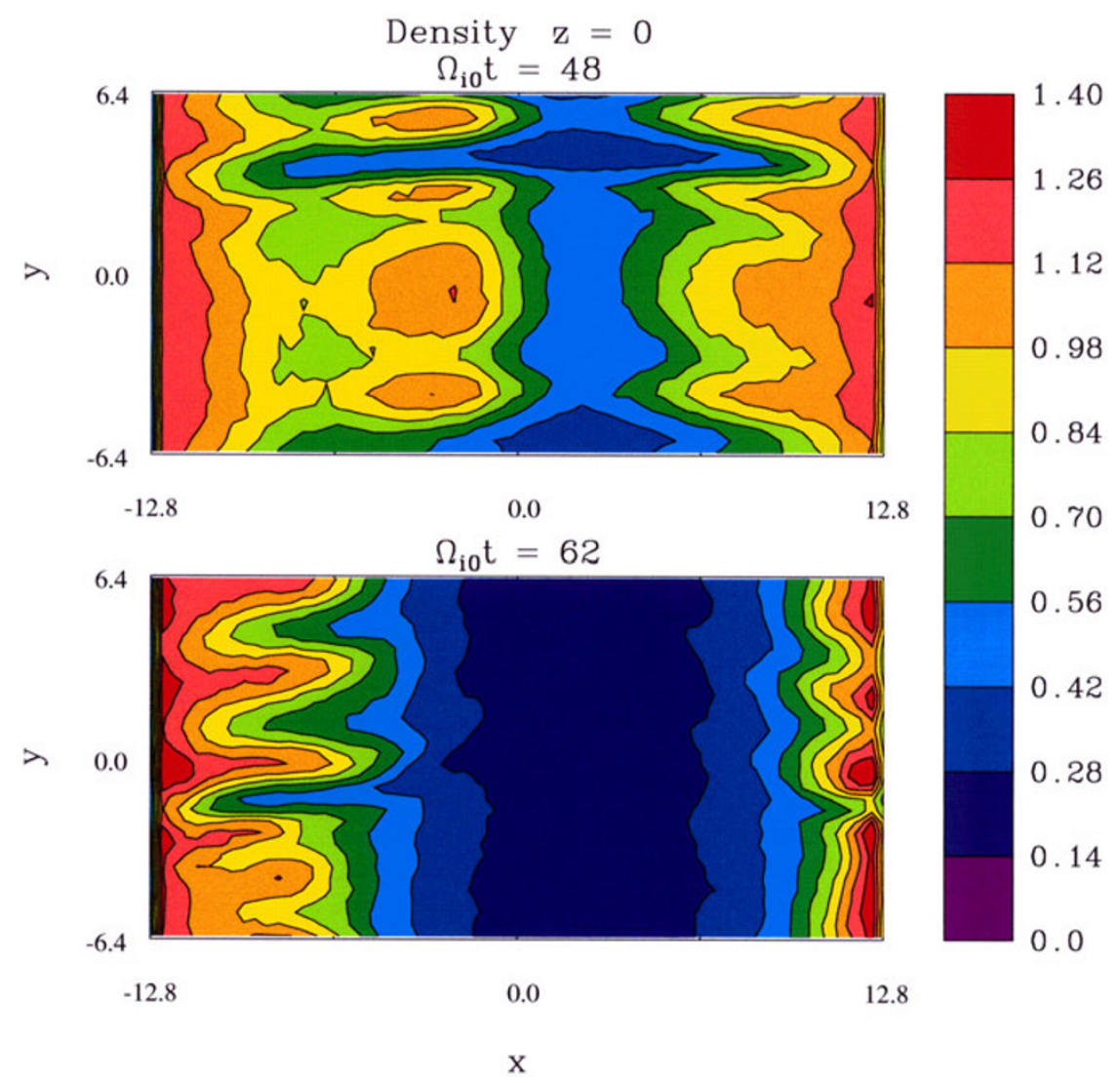

Fig. 3. Contour plots of the density in the $x, y$ plane at $z=0$ at times $\Omega_{i 0} t=48$ and 62 for a 3-D simulation of the Harris neutral sheet with an initial magnetic island perturbation.

is applied at the boundaries as described in Section 4. This represents the field imposed on the magnetotail by the solar wind.

Typical parameters for the first configuration (Section 3) are a system size of $-12.8 c / \omega_{p i} \leq x \leq 12.8 c / \omega_{p i}$ and $-6.4 c / \omega_{p i} \leq y, z \leq 6.4 c / \omega_{p i}$, where the ion skin depth $c / \omega_{p i}$ is computed using the peak density $n_{0}$. Additional parameters are $m_{i} / m_{e}=25, T_{e} / T_{i}=0.2, n_{b} / n_{0}=0.2$, and $c / \omega_{p i}=10 \Delta$, where $\Delta$ is the grid spacing. Some $25-30 \times$ $10^{6}$ particles are used for each species. For the magnetotail configuration (Section 4 ) the mass and temperature ratios are altered to $m_{i} / m_{e}=16$ and $T_{e} / T_{i}=0.25$, and the system grid is changed to $N_{x} \times N_{y} \times N_{z}=128 \times 256 \times 192$.

\section{Reconnection in a Current Sheet}

The structure of the diffusion region in collisionless reconnection and the factors controlling the rate of reconnection have been examined in a large number of 2-D PIC simulations in recent years (see the references in Pritchett, 2000). When the third dimension is added, a new class of finite- $k_{y}$ instabilities can be excited which may modify the structure of the diffusion region. Of these new modes, the drift kink mode (Zhu and Winglee, 1996; Ozaki et al., 1996; Pritchett et al., 1996) has received considerable attention. This mode is driven by the relative ion-electron drift parallel to the initial current, and it results in a kinking or firehose displacement of the current sheet in $z$. For small values of the ion to electron mass ratio $\left(m_{i} / m_{e} \lesssim 25\right)$ the growth rate is up to a factor of two faster than for the tearing instability. Figure 1 shows results from a 3-D simulation comparing the time histories of the pure kink modes $\left(k_{x}=0\right.$, finite $\left.k_{y}\right)$ and the pure tearing modes (finite $k_{x}, k_{y}=0$ ). In this simulation the half-thickness of the current sheet is $L=c / \omega_{p i}$, and the normal field parameter is $\epsilon=0.04$. The kink mode 2 with wavenumber $k_{y} L=0.98$ dominates the evolution of the current sheet. It reaches saturation at a time $\Omega_{i 0} t \sim 50$ 60 , well before the tearing modes exhibit significant growth. The displacements in $z$ produced by the kink mode are of the order of 1-2 $L$, and thus they do not completely destroy the current sheet (Fig. 2). Also, the perturbed ion velocities are much smaller than those for the electrons (by roughly a factor of $\left.\left(m_{e} / m_{i}\right)^{1 / 2}\right)$, and consequently the cross tail ion flow and current are not strongly modified. At later times the 2-D tearing modes with $k_{x} L \sim 0.3-0.6$ do grow to substantial levels at roughly the same rate as in the strictly 2-D $(x, z)$ case. This simulation shows no evidence for a "sausage" type instability such as has been reported by Büchner and Kuska (1999), nor is there any clear evidence for growth of the lower hybrid drift instability. This latter mode is presumably stabilized by the finite $k_{\|}$introduced by the initial normal field component (Gladd, 1976).

A complete Vlasov analysis of the linear stability of the drift kink mode has now been completed by Daughton (1998, 1999). He has shown that the dominance of the drift kink mode relative to the tearing mode is an artifact resulting from the use of the small $m_{i} / m_{e}$ ratio. The drift kink growth rate 


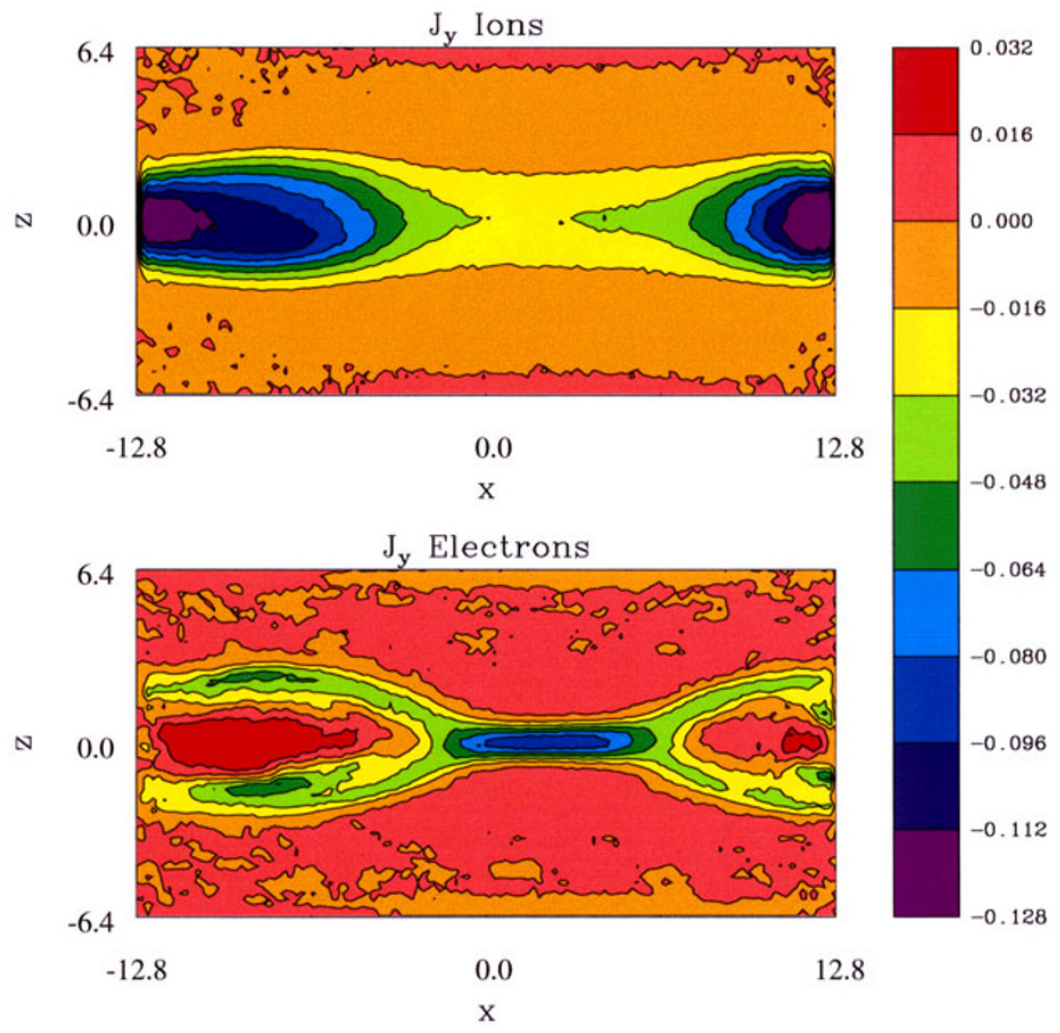

Fig. 4. The current density $J_{y}(x, z)$ averaged over all $y$ for the ions and electrons at time $\Omega_{i 0} t=62$ for the same simulation as in Fig. 3 .
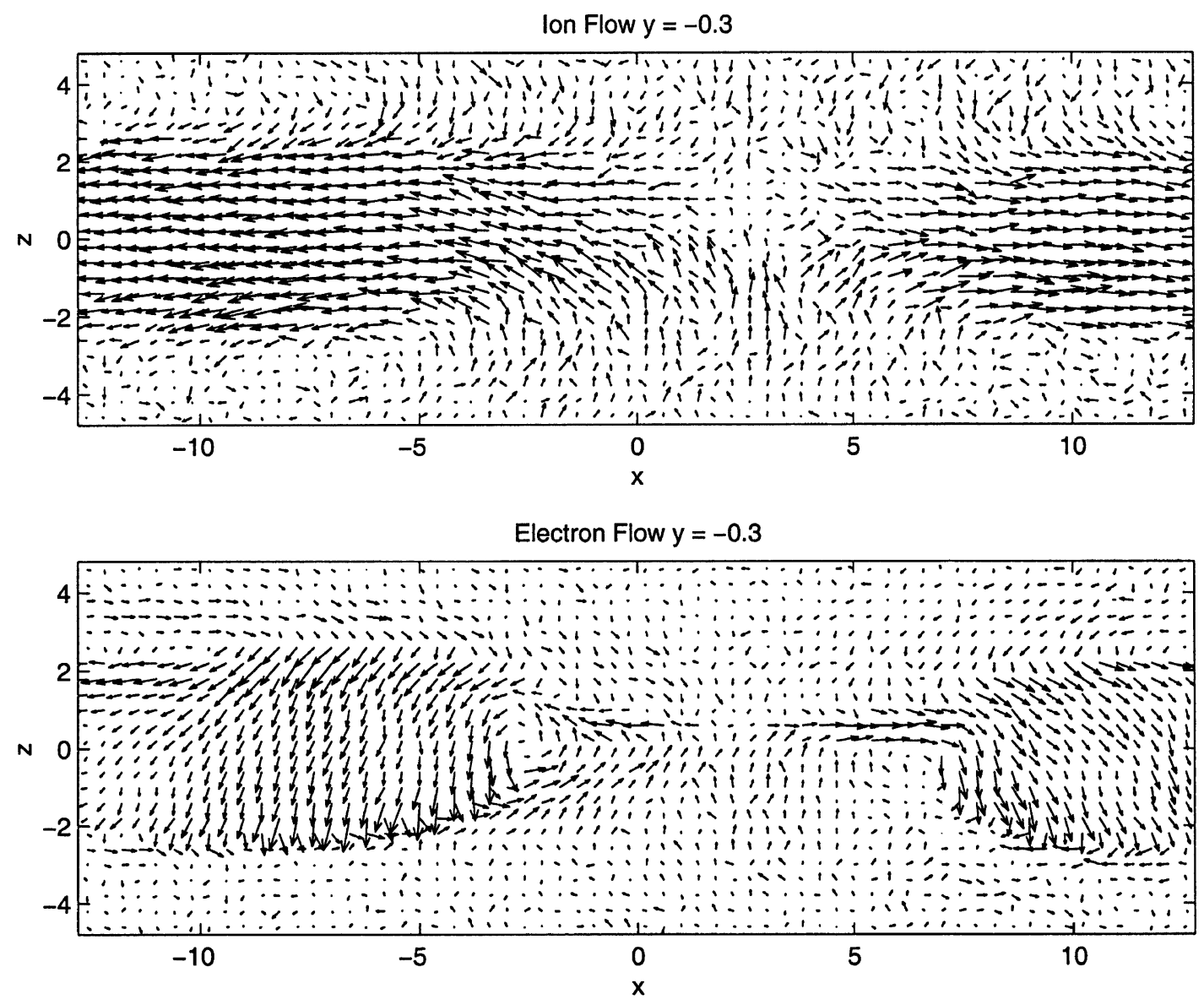

Fig. 5. Ion and electron bulk flow velocities in the $x, z$ plane at $y=-0.3 c / \omega_{p i}$ at time $\Omega_{i 0} t=62$ for the same simulation as in Fig. 3. The peak ion flow speed is $0.49 v_{A}$, while the peak electron speed is $2.15 v_{A}$. 


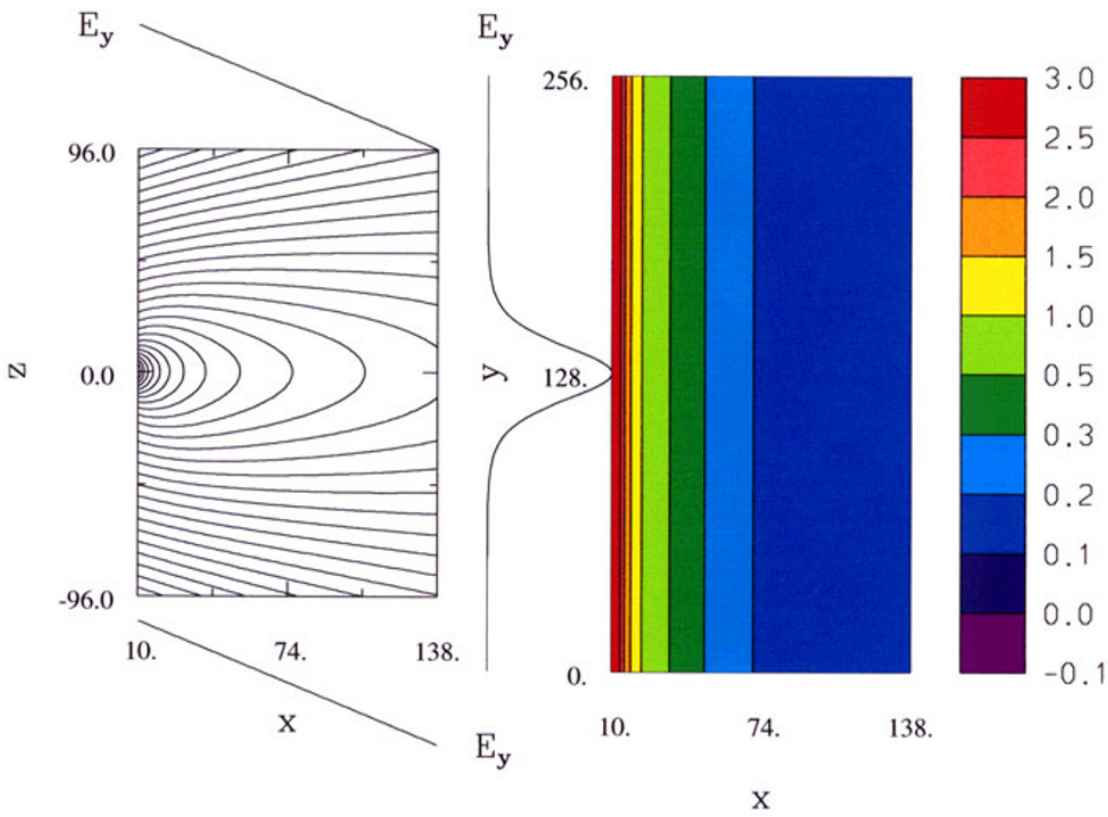

Fig. 6. Initial magnetic field configuration for the magnetotail simulations. The left panel shows the magnetic field lines in a meridian plane and the magnitude of the driving electric field $E_{y}$ applied at the lobe boundaries. The right panel shows the strength of the magnetic field in the equatorial plane and of the driving electric field applied at the inner $x$ boundary.
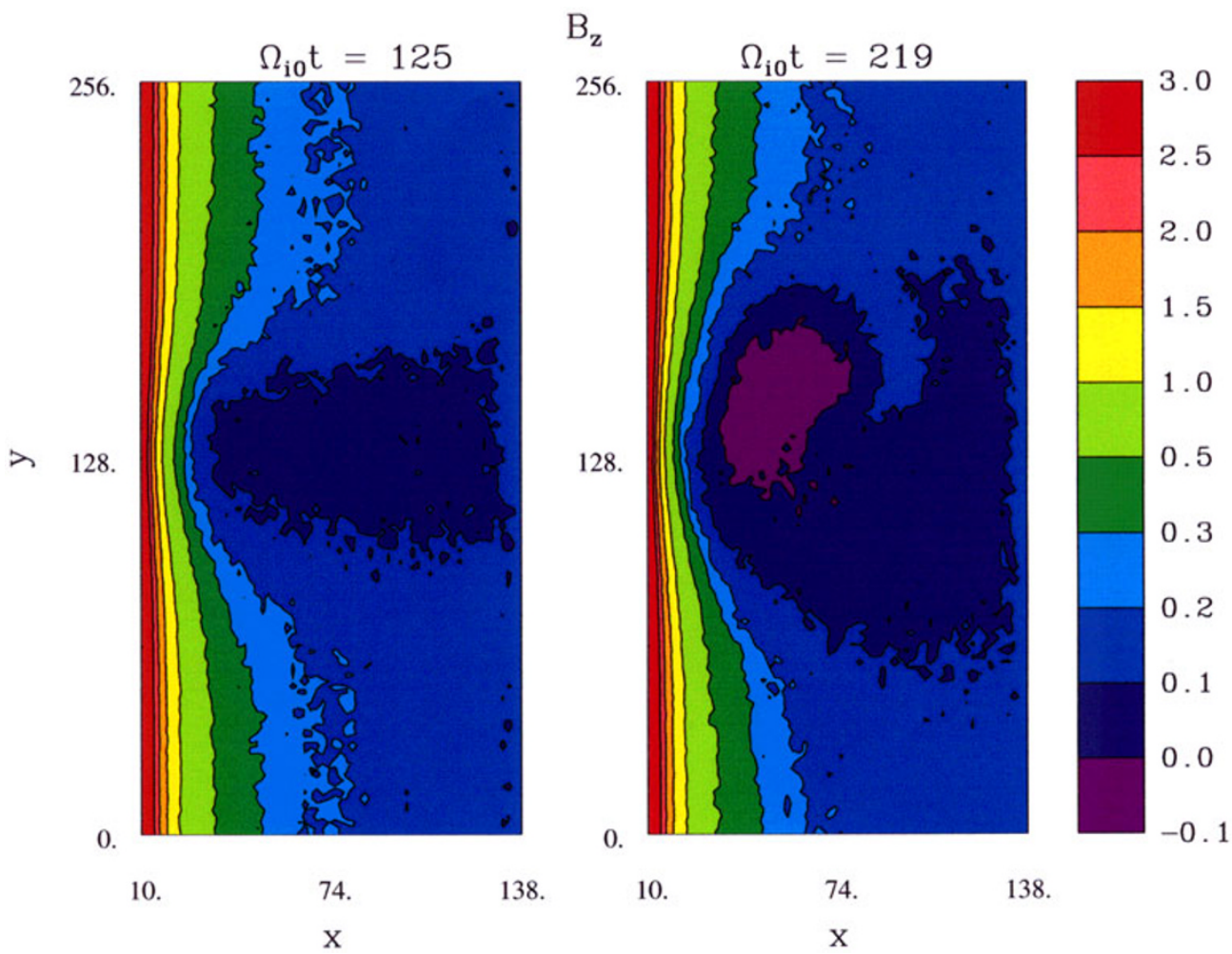

Fig. 7. Contour plots of the magnetic field in the equatorial plane at times $\Omega_{i 0} t=125$ and $\Omega_{i 0} t=219$ in the magnetotail simulation.

decreases rapidly as $m_{i} / m_{e}$ is increased. Equality with the tearing growth rate occurs for $m_{i} / m_{e} \approx 80$, and for the proton/electron ratio of 1836 the kink growth rate is smaller by a factor of $\sim 20$. This rapid fall off is a consequence of the reduced overlap of the antisymmetric $E_{y}(z)$ drift kink eigenfunction (whose spatial scale is set by the current half width $L \sim c / \omega_{p i}$ ) with the electron spatial scale as $m_{e} / m_{i}$ is decreased. This predicted decrease of the kink growth rate with increasing $m_{i} / m_{e}$ has been verified by 2-D PIC simulations for values of $m_{i} / m_{e}$ up to 100 (Hesse and Birn, 2000).

In order to examine the process of 3-D reconnection when 

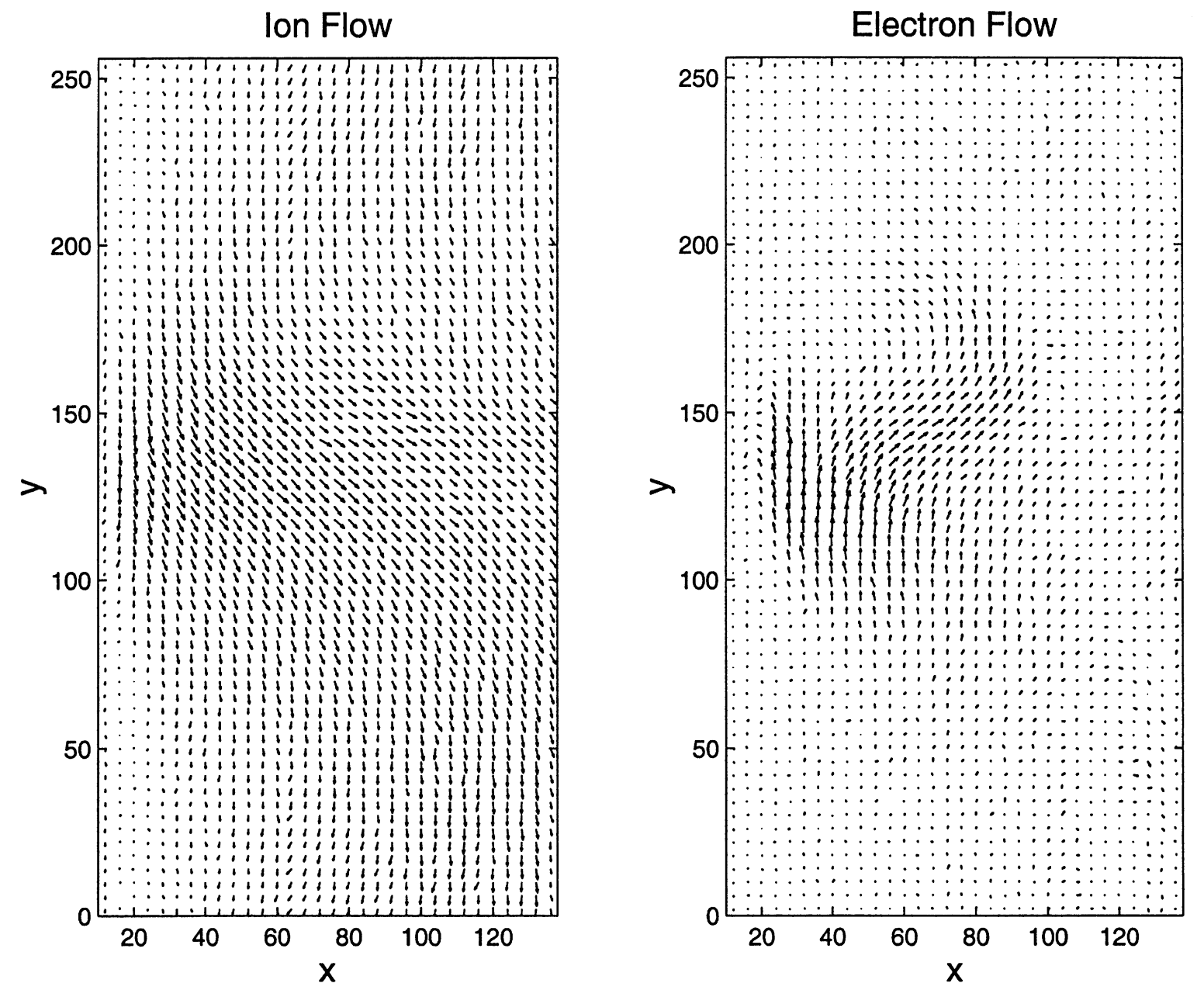

Fig. 8. Ion and electron flow velocities in the equatorial plane at time $\Omega_{i 0} t=219$ for the same simulation as in Fig. 7 . The peak ion flow speed is $0.25 v_{T i}$, while the peak electron speed is $0.51 v_{T i}$.

the drift kink mode is not completely dominant, we have performed an additional simulation in which a perturbation that creates an $X$ line is imposed at $t=0$,

$$
\psi(x, z)=\psi_{0} \cos \left(\pi x / L_{x}\right) \cos \left(\pi z / L_{z}\right) .
$$

This is analogous to the procedure used in the 2-D GEM magnetic reconnection challenge problem (Birn et al., 2001); the net effect is to put the system in the nonlinear regime of magnetic reconnection prior to the growth of the drift kink mode. The subsequent evolution of the system is illustrated in Fig. 3, which shows the density in the $z=0$ plane at two different times. At the earlier time $\left(\Omega_{i 0} t=48\right)$ the expulsion of plasma away from the neutral line accompanying reconnection has begun to lower the density in the diffusion region near $x=0$, while the kink mode has again created a modulation in the density as a function of $y$. In the region of strongest nonlinear development of the kink mode $\left(y \approx 4 c / \omega_{p i}\right)$, the density variation is coherent across almost the entire system; otherwise it tends to be localized in the outflow regions. At the later time $\left(\Omega_{i 0} t=62\right)$, the 2-D reconnection has produced a strong reduction in the density in the central portion of the plane for all values of $y$. The low density background plasma which now dominates the central region has no relative ion-electron drift and cannot support the kink mode; the kink perturbations are then swept out of the system.

Figure 4 shows the $J_{y}$ current densities averaged over all values of $y$ at time $\Omega_{i 0} t=62$ for the ions and electrons. This plot shows that the characteristic thin electron current layer with vertical scale size of a few $c / \omega_{p e}$ is formed in the 3-D case as well. Here the electron current dominates over that for the ions, despite the much higher initial ion temperature. Figure 5, which shows the bulk ion and electron velocities in the $x, z$ plane at $y=-0.3 c / \omega_{p i}$, indicates that the inflowing ions become demagnetized at distances of a few $c / \omega_{p i}$ away from the neutral line and then are accelerated toward the outflow boundary, reaching a speed of $\sim 0.5-0.6 v_{A}$. The electron flows are strongly perturbed by the kink mode in the outflow region. In Fig. 5, the electron $v_{z}$ flow is mainly negative, whereas at a half wavelength away, the flow is mainly positive. On the average, however, the differences between the electron and ion flows in the $x, z$ plane (Hall effect) produce the characteristic quadrupolar $B_{y}$ field as observed in 2-D treatments of reconnection.

It thus appears that the reconnection process for a current sheet in 3D is quite similar to the familiar 2-D case. For a realistic $m_{i} / m_{e}$ value, the drift kink mode is not likely to play an important role, except perhaps for modifying the electron flow velocities. The reconnection rate is mainly 


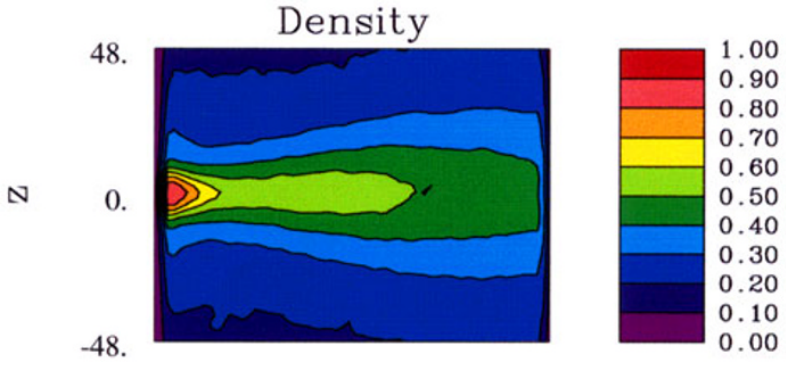

10.

74.

138.

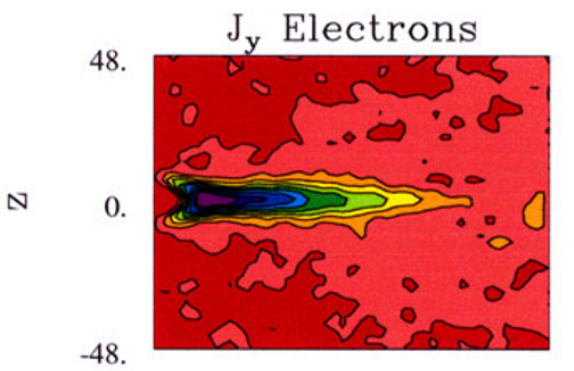

10.

74.

138.

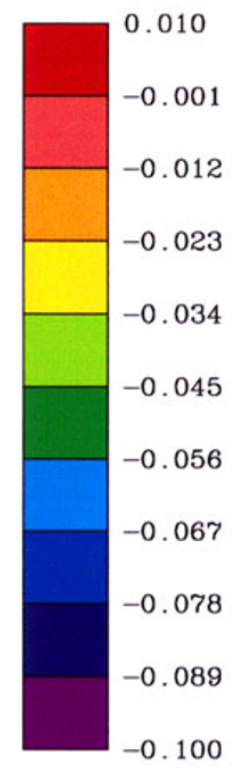

-48 .

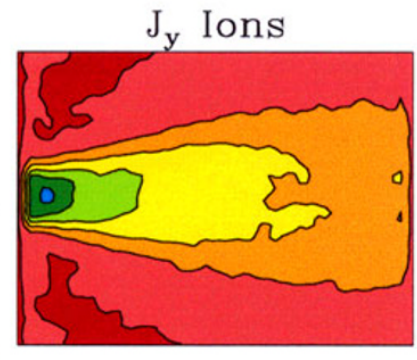

10.

74.

138.

$\mathrm{X}$

Fig. 9. Contour plots of the density, electron current density $J_{y}$, and ion current density $J_{y}$ in the $x, z$ plane averaged over the spatial interval $112 \leq y / \Delta \leq 144$ at time $\Omega_{i 0} t=219$ for the same simulation as in Fig. 7 .

sensitive to whistler dynamics in the region outside of the thin electron layer, and this physics does not seem to be modified significantly in 3D.

\section{Driven Reconnection in a 3-D Model of the Mag- netotail}

Figure 6 shows the initial magnetic field configuration for the magnetotail simulations. The left panel shows the magnetic field lines in a meridian plane. The line dipole is located at $x=0$, and the simulation region extends from $x / \Delta=10$ to $x / \Delta=138$. The right panel shows the strength of the magnetic field in the equatorial plane. This varies from $5.1 B_{0}$ at the left boundary to $0.13 B_{0}$ at the right boundary, where $B_{0} \sim 20 \mathrm{nT}$ is a typical lobe field strength. This variation in $B_{z}$ corresponds roughly to the range from $7 \mathrm{R}_{\mathrm{E}}$ to $20 \mathrm{R}_{\mathrm{E}}$ in the tail. The initial plasma sheet half thickness is relatively large, with $L / \rho_{i 0} \approx 4$, where $\rho_{i 0}$ is the ion gyroradius in the lobe field.

To model the effect of the convection electric field imposed by the solar wind on the tail, we impose a boundary field

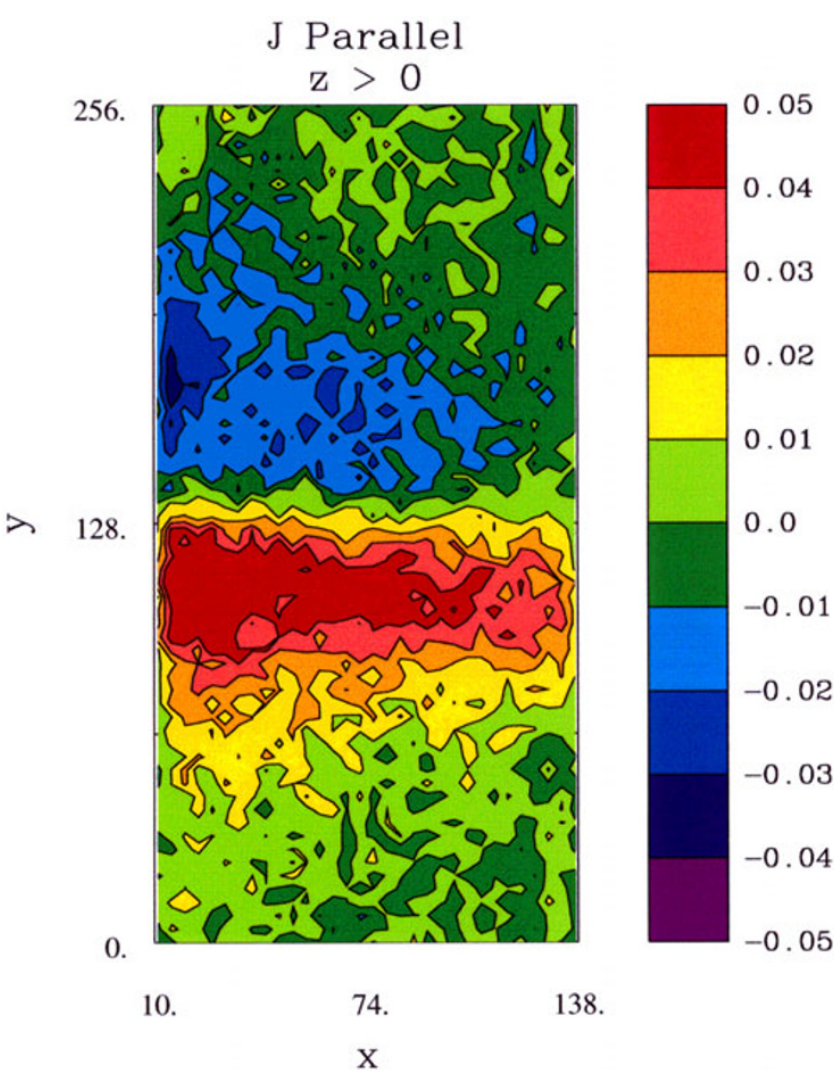

Fig. 10. Contour plot at time $\Omega_{i 0} t=219$ of the field-aligned current density $J_{\|}(x, y)$ integrated over all $z>0$.

$E_{y}$ in the lobes and at the inner boundary. As indicated schematically in Fig. 6, the strength of this field decreases linearly with increasing distance $x$. As a function of $y$, the field has a sech ${ }^{2}$ dependence, with the maximum occurring at $y / \Delta=128$; this defines midnight in the simulation. Values of $y / \Delta>128$ then correspond to the dawn side of the tail, and values of $y / \Delta<128$ correspond to the dusk side. This driving field is directed from dawn to dusk, and it is turned on smoothly at the beginning of the simulation over a period of $\Omega_{i 0} t \sim 5$, roughly a full ion gyroperiod. The peak driving field has a strength $\left(c / v_{T i}\right) E_{0 y} / B_{0} \approx 0.08$, where $v_{T i}=$ $\left(2 T_{i 0} / m_{i}\right)^{1 / 2}$ is the ion thermal speed.

The effect of the inductive field at the inner boundary is to pull flux out of the simulation region. Thus the equatorial magnetic field is locally reduced. This is illustrated in Fig. 7. As shown in the left panel $\left(\Omega_{i 0} t=125\right)$, the contours of $B_{z}$ are pulled closer to the dipole region near midnight; as a consequence of the flux depletion, the field in the mid-tail region is reduced. As this process continues, the equatorial field is driven to reverse sign, i.e., to reconnect, in a localized region stretching dawnward from midnight (right panel, $\Omega_{i 0} t=219$ ). At still later times this southward $B_{z}$ region continues to expand dawnward and tailward. As the reconnection field region develops, the magnetic field lines locally become ever more stretched and then develop a spiral structure resembling a flux rope.

The cross tail current becomes locally enhanced in the region of decreasing field strength. This is to be expected since pressure balance requires that $J_{y} B_{z} / c=\partial p / \partial x$, and 
the pressure gradient does not change appreciably. This enhanced current is primarily carried by the electrons. Figure 8 shows the equatorial flow velocities of the ions and electrons at $\Omega_{i 0} t=219$. The ion flow is enhanced and diverted tailward in the reconnection region; the peak speed is $0.25 v_{T i}$. The electron flow is strongly localized between $y / \Delta \approx 100$ and $y / \Delta \approx 150$ and is diverted tailward in the dawn portion of this region; the peak speed is $0.51 v_{T i}$.

Figure 9 shows density and current distributions in the meridian plane averaged over the region $112 \leq y / \Delta \leq 144$. It is apparent that the enhanced electron current is contained in a thin current sheet within the much broader plasma sheet determined by the density and ion $J_{y}$ distributions. At later times, the higher density plasma at the center of the sheet tends to expand tailward on the dawn side of midnight, while lower density plasma moves earthward on the dusk side of midnight. The electron current region then extends further tailward, but it is broader and has a smaller magnitude in this region.

The enhanced equatorial current near midnight is in fact connected to a field-aligned current system. Figure 10 shows the parallel current integrated over all positive values of $z$ projected onto the $x, y$ plane. (Integration over all $z<0$ gives a plot with a reversed sign.) The color coding is such that blue corresponds to a current out of the ionosphere and into the tail, while yellow-red corresponds to a current out of the tail and into the ionosphere. Thus there is a region 2 type current system whose elements are located on either side of midnight; this current system feeds the enhanced current in the reconnection region. It has the opposite sense to the region 1 current system associated with the current wedge and the reduction in cross-tail current associated with substorm onset.

\section{Summary and Discussion}

We have used 3-D PIC simulations to make some initial studies of the effects on collisionless reconnection of the new processes present in a fully 3 -D configuration. For the idealized current sheet of the Harris type, it appears that the drift kink mode will not play a significant role for a realistic value of the ion to electron mass ratio. In the absence of this mode, the tearing instability appears to develop quite similarly to the well-investigated 2-D case. The diffusion region develops a multiscale structure based on electron and ion scale lengths (Biskamp et al., 1997; Shay et al., 1998). Within a distance of the order of $c / \omega_{p i}$, the ion motion decouples from that of the electrons, and the ions are accelerated away from the $X$ line. The electrons remain frozen-in to the magnetic field down to the scale of $c / \omega_{p e}$. Within this region the electrons are strongly accelerated by the induction electric field $E_{y}$, and they are the dominant carrier of the cross-tail current $J_{y}$. Previous studies have shown that the reconnection rate is essentially independent of the specific mechanism which breaks the frozen-in condition in this inner region (Shay and Drake, 1998; Birn et al., 2001).

A 2-D tearing instability, however, cannot account for the localized onset of substorms in the geomagnetic tail. We performed a 3-D simulation in which a localized convection electric field was applied at the lobe and near-Earth boundaries. This resulted in a localized reduction in the equato- rial magnetic field and a stretching of the field lines. This process was accompanied by the formation of an embedded thin current sheet where the electrons are the dominant current carrier. This thin region of enhanced cross-tail current is connected to a type 2 field-aligned current system. If the driving process continues, localized reconnection occurs with the formation of a region of southward $B_{z}$. This region of increased density and southward $B_{z}$ then expands tailward. This simulation fails, however, to reproduce the substorm current wedge phenomenon which involves a diversion of part of the cross-tail current into the ionosphere, and thus its relevance to substorm onset is ambiguous at best.

Acknowledgments. This work was supported by NASA grants NAG 5-4339 and NAG 5-8132. The particle simulations were performed at the National Energy Research Scientific Computing Center at the Lawrence Berkeley National Laboratory and at the San Diego Supercomputer Center, which is supported by the National Science Foundation.

\section{References}

Angelopoulos, V., T. D. Phan, D. E. Larson, F. S. Mozer, R. P. Lin, K Tsuruda, H. Hayakawa, T. Mukai, S. Kokubun, T. Yamamoto, D. J. Williams, R. W. McEntire, R. P. Lepping, G. K. Parks, M. Brittnacher, G. Germany, J. Spann, H. J. Singer, and K. Yumoto, Magnetotail flow bursts: Association to global magnetospheric circulation, relationship to ionospheric activity and direct evidence for localization, Geophys. Res. Lett., 24, 2271-2274, 1997.

Birn, J., R. Sommer, and K. Schindler, Open and closed magnetospheric tail configurations and their stability, Astrophys. Space Sci., 35, 389-402, 1975.

Birn, J., J. F. Drake, M. A. Shay, B. N. Rogers, R. E. Denton, M. Hesse, M. Kuznetsova, Z. W. Ma, A. Bhattacharjee, A. Otto, and P. L. Pritchett, GEM magnetic reconnection challenge, J. Geophys. Res., 106, 37153719, 2001

Biskamp, D., E. Schwarz, and J. F. Drake, Two-fluid theory of collisionless magnetic reconnection, Phys. Plasmas, 4, 1002-1009, 1997.

Büchner, J. and J.-P. Kuska, Sausage mode instability of thin current sheets as a cause of magnetospheric substorms, Ann. Geophys., 17, 604-612, 1999.

Daughton, W., Kinetic theory of the drift kink instability in a current sheet, J. Geophys. Res., 103, 29,429-29,443, 1998.

Daughton, W., The unstable eigenmodes of a neutral sheet, Phys. Plasmas, 6, 1329-1343, 1999.

Gladd, N. T., The lower hybrid drift instability and the modified two stream instability in high density theta pinch environments, Plasma Phys., 18, 27-40, 1976.

Hesse, M. and J. Birn, Near- and mid-tail current flow during substorms: Small- and large-scale aspects of current disruption, in Magnetospheric Current Systems, Geophys. Monogr. Ser., Vol. 118, edited by S. Ohtani, R. Fujii, M. Hesse, and R. L. Lysak, pp. 295-303, AGU, Washington, D.C., 2000.

Lembège, B. and R. Pellat, Stability of a thick two-dimensional quasineutral sheet, Phys. Fluids, 25, 1995-2004, 1982.

Lui, A. T. Y., K. Liou, P. T. Newell, C. I. Meng, S. I. Ohtani, T. Yamamoto, T. Ogino, S. Kokubun, M. J. Brittnacher, and G. K. Parks, Plasma sheet behavior associated with auroral breakups, in Substorms-4, edited by S. Kokubun and Y. Kamide, pp. 183-186, Kluwer Acad. Publ., Dordrecht, 1998

Ozaki, M., T. Sato, R. Horiuchi, and the Complexity Simulation Group, Electromagnetic instability and anomalous resistivity in a magnetic neutral sheet, Phys. Plasmas, 3, 2265-2274, 1996.

Petrukovich, A. A., V. A. Sergeev, L. M. Zelenyi, T. Mukai, T. Yamamoto, S. Kokubun, K. Shiokawa, C. S. Deehr, E. Y. Budnick, J. Büchner, A. O Fedorov, V. P. Grigorieva, T. J. Hughes, N. F. Pissarenko, S. A. Romanov, and I. Sandahl, Two spacecraft observations of a reconnection pulse during an auroral breakup, J. Geophys. Res., 103, 47-59, 1998.

Pritchett, P. L., Particle-in-cell simulations of magnetosphere electrodynamics, IEEE Trans. Plasma Sci., 28, 1976-1990, 2000.

Pritchett, P. L. and F. V. Coroniti, Formation of thin current sheets during plasma sheet convection, J. Geophys. Res., 100, 23,551-23,566, 1995. 
Pritchett, P. L. and F. V. Coroniti, Interchange instabilities and localized high-speed flows in the convectively-driven near-Earth plasma sheet, in Substorms-4, edited by S. Kokubun and Y. Kamide, pp. 443-448, Kluwer Acad. Publ., Dordrecht, 1998.

Pritchett, P. L., F. V. Coroniti, and V. K. Decyk, Three-dimensional stability of thin quasi-neutral current sheets, J. Geophys. Res., 101, 27,41327,429, 1996.

Pritchett, P. L., F. V. Coroniti, and R. Pellat, Convection-driven reconnection and the stability of the near-Earth plasma sheet, Geophys. Res. Lett., 24, 873-876, 1997.

Schindler, K., A self-consistent theory of the tail of the magnetosphere, in Earth's Magnetospheric Processes, edited by B. M. McCormac, pp. 200209, D. Reidel, Norwood, Mass, 1972.

Shay, M. A. and J. F. Drake, The role of electron dissipation on the rate of collisionless magnetic reconnection, Geophys. Res. Lett., 25, 3759-3762, 1998.
Shay, M. A., J. F. Drake, R. E. Denton, and D. Biskamp, Structure of the dissipation region during collisionless magnetic reconnection, J. Geophys. Res., 103, 9165-9176, 1998.

Sonnerup, B. U. Ö., Magnetic Field Reconnection, in Solar System Plasma Physics, Vol. III, edited by L. T. Lanzerotti, C. F. Kennel, and E. N. Parker, pp. 45-108, North-Holland, Amsterdam, 1979.

Terasawa, T., Hall current effect on tearing mode instability, Geophys. Res. Lett., 10, 475-478, 1983.

Zhu, Z. and R. M. Winglee, Tearing instability, flux ropes, and the kinetic current sheet kink instability in the Earth's magnetotail: A threedimensional perspective from particle simulations, J. Geophys. Res., 101, 4885-4897, 1996

P. L. Pritchett (e-mail: pritchet@physics.ucla.edu) and F. V. Coroniti 